\title{
Simple and Fast Detection of Homocysteine by cucurbit[7]uril Fluorescent Probe based on Competitive Strategy
}

\section{Chenrui Jiang}

China Pharmaceutical University

\section{Deli Xiao}

China Pharmaceutical University

\section{Ping Yang}

China Pharmaceutical University

Wenyao Tao

China Pharmaceutical University

\section{Zhaorui Song}

China Pharmaceutical University

Hua He ( $\nabla$ jcb_321@163.com )

China Pharmaceutical University https://orcid.org/0000-0002-4983-6250

\section{Research Article}

Keywords: Supramolecular probe, Host-guest interaction, Competitive strategy, Homocysteine detection

Posted Date: October 25th, 2021

DOI: https://doi.org/10.21203/rs.3.rs-974718/v1

License: () (1) This work is licensed under a Creative Commons Attribution 4.0 International License. Read Full License

Version of Record: A version of this preprint was published at Chemical Physics Letters on January 1st, 2022. See the published version at https://doi.org/10.1016/j.cplett.2022.139382. 
Simple and fast detection of homocysteine by cucurbit[7]uril fluorescent probe based on competitive strategy

Chenrui Jiang ${ }^{\text {a,+}}$, Deli Xiao ${ }^{\text {a, c, d,+ }}{ }^{\text {, Ping Yang a }}{ }^{\text {, Wenyao Tao }}{ }^{\text {a }}$, Zhaorui Song ${ }^{\text {a, b,* }}$, Hua $\mathrm{He}^{\mathrm{a}, \mathrm{c}, \mathrm{d}, *}$

${ }^{a}$ Department of Analytical Chemistry, China Pharmaceutical University, Nanjing, 211198, China

b Bohai rim Advanced Research Institute for Drug Discovery, Yantai, 264003, Shandong, China

c Key Laboratory of Biomedical Functional Materials, China Pharmaceutical University, Nanjing, 211198, China

d Key Laboratory of Drug Quality Control and Pharmacovigilance, Ministry of Education, China Pharmaceutical University, Nanjing, 211198, China

+ These authors contributed equally to this work.

* Corresponding author.

Tel: 025-83271509-588(O), Fax: 025-83271505.

E-mail addresses: jcb_321@163.com, dochehua@163.com (Hua. He), songzhaorui1016@163.com (Zhaorui Song).

\section{Abstract}

This research put forward a simple and rapid fluorescence probe method based on competition strategy to detect homocysteine. The host-guest interaction behavior between cucurbit[7]uril and homocysteine was further studied via UV-vis spectroscopy, Fourier transform infrared spectroscopy, molecular modeling, and ${ }^{1} \mathrm{H}$ NMR spectroscopy in aqueous solution. The binding constant of cucurbit[7]uril-homocysteine measured by ${ }^{1} \mathrm{H}$ NMR titration is $2.40 \times 10^{3} \mathrm{~L} \mathrm{~mol}^{-1}$. Under the optimal detection conditions, the fluorescence intensity of the cucurbit[7]uril-berberine complex declined linearly with increasing concentration of homocysteine in the range from 5 to $200 \mu \mathrm{M}$, and the limit of detection was $1.76 \mu \mathrm{M}$. This established fluorescent probe method exhibits high selectivity for coexisting interfering substances. Meanwhile, it was also successfully employed to detect homocysteine in serum samples with the recovery rate of $85.1 \%$ to $102.0 \%$. This supramolecular fluorescent probe based on competitive strategy provides a simple and fast method for the determination of homocysteine in practical samples.

Keywords: Supramolecular probe $\cdot$ Host-guest interaction - Competitive strategy · 
Homocysteine detection

\section{Introduction}

Homocysteine (Hcy), as a reactive sulfhydryl-comprising amino acid, is an important intermediate creation during the metabolism of methionine and cysteine, which can be released into the blood $[1,2]$. The plasma of a normal adult can contain 5-15 $\mu \mathrm{M}$ homocysteine [3, 4]. Moreover, hyperhomocysteinemia (HHcy) is characterized by the irregular increase of homocysteine concentration in plasma. HHcy is classified into mild $(16-30 \mu \mathrm{M})$, modest $(31-100 \mu \mathrm{M})$ and severe $(>100 \mu \mathrm{M})$ according to elevated concentrations of total Hcy [2]. Abnormal Hcy levels can cause a variety of diseases, such as cardiovascular diseases, neurological diseases, osteoporosis, inflammatory bowel disease, and complications with pregnancy $[5,6]$.

So far, a great number of methods have been reported for detecting Hcy, including radioenzyme immunoassay (RIA) [7], high performance liquid chromatography (HPLC) [8-13], electrochemistry [2, 14], capillary electrophoresis (CE) $[15,16]$, colorimetry [1, 17-20], liquid chromatography-mass spectrometry (LC-MS) [21-23], etc. [24]. However, radioenzyme immunoassay utilizes radioactive adenosine, which can cause pollution and require expensive antibody costs. The instrumental analysis has some inevitable limitations such as tedious sample pretreatment, complicated chemical modification, precise instruments, long analysis time and low sensitivity [25]. Compared with the above analytical methods, the fluorescence probe method has attracted extensive concern owing to its rapidness, simplicity, high sensitivity, good stability, and low cost. Therefore, it is extremely indispensable to develop a convenient, fast, sensitive and stable fluorescence probe method for the determination of Hcy content in practical applications.

Cucurbit[n]urils $(\mathrm{CB}[\mathrm{n}], \mathrm{n}=5-8,10)$ are the macrocyclic molecular containers, which are consisted of $n$ glycoluril monomers linked with $2 \mathrm{n}$ methylene groups. Symmetrical $\mathrm{CB}[\mathrm{n}]$ hosts have hydrophobic cavities and restrictive polar uridylcarbonyl portals [26, 27]. $\mathrm{CB}[\mathrm{n}]$ and its derivatives can combine with various guest molecules through hydrogen bonding, hydrophobic interaction, and ion-dipole interaction $[26,28,29]$. Guest molecules are encapsulated in the cavity of $\mathrm{CB}[\mathrm{n}]$ via specific host-guest interactions. Supramolecular complex usually alters the photophysical and photochemical properties of the contained guest molecules [29, 30]. Owing to its excellent solubility in aqueous solutions and the appropriate cavity size, $\mathrm{CB}[7]$ has received special attention in fluorescence analysis in recent years. In 
addition, $\mathrm{CB}$ [7] possesses the extraordinary competency to constitute host-guest complexes with organic compound and cations [28, 31].

In this study, a fluorescent probe method based on the competitive strategy has been developed to detect Hcy in practical samples. The host-guest interaction mechanism and binding constant between the $\mathrm{CB}[7]$ and Hcy have also been investigated. On the basis of host-guest recognition and concentration advantage, Hcy can occupy the hydrophobic cavity of $\mathrm{CB}[7]$ by competing with berberine hydrochloride (BER), resulting in a significant change in the fluorescence spectrum. The proposed method can achieve simple, rapid and highly selective determination of Hcy without expensive equipment and complicated operations.

\section{Experimental}

\section{Materials and reagents}

The homocysteine (Hcy) was obtained from Shanghai Hanhong Technology Co., Ltd. Berberine hydrochloride (BER) was purchased from Nanjing Dulai Biotechnology Co., Ltd. Cucurbit[7]uril (CB[7]) was acquired from Shanghai Nafu Biotechnology Co., Ltd. All other chemicals were analytical reagent grade, and double-distilled water was worked through the whole of the process.

\section{Apparatus}

Fluorescence spectra (FL) were recorded using a RF-5301PC fluorescence spectrophotometer (Shimadzu, Japan). Fourier-transform infrared spectroscopy (FT-IR) was acquired by a FT-IR 8400s Fourier-transform infrared spectrophotometer (Shimadzu, Japan). Nuclear magnetic resonance spectroscopy $\left({ }^{1} \mathrm{H}\right.$ NMR) was performed on a Bruker Avance-500 (500 MHz, Bruker, Switzerland). Ultraviolet-visible (UV-vis) absorption spectra were recorded through a UV-1800 UV-vis spectrophotometer (Shimadzu, Japan). All optical records were measured under stable ambient conditions.

\section{Spectra measurement procedure}

The fluorescence measurement was performed at an excitation wavelength of $343 \mathrm{~nm}$ in the absence or presence of Hcy solution. $2 \mathrm{~mL} \mathrm{CB}$ [7]-BER $(3 \mu \mathrm{M}: 2 \mu \mathrm{M})$ standard stock solution was set in a $4 \mathrm{~mL}$ EP tube. Subsequently, a given concentration of Hcy aqueous solution was added in sequence. Afterwards the mixture was shaken fully for 15 minutes at room temperature. Finally, the fluorescence change values $\left(\mathrm{F} / \mathrm{F}_{0}=\mathrm{F}_{\mathrm{CB}[7]-\mathrm{BER}+\mathrm{Hcy}} / \mathrm{F}_{\mathrm{CB}[7]-\mathrm{BER})}\right)$ were calculated. 


\section{Examination of spiked bovine serum}

The fluorescent probe based on CB[7] was applied to determine Hcy in actual serum samples. Disparate concentrations of Hcy were appropriately subjoined to the diluted bovine serum samples, and then the spectroscopic method described in Section 2.3 was performed.

\section{Results and discussion}

\section{Characterization of CB[7]-Hcy inclusion complex}

\section{UV-vis spectroscopy}

First, UV-vis technology was utilized to explore the formation of the CB[7]-Hcy system in aqueous solution. The trial consequence was shown in Fig. S1, BER displayed absorption peaks at $228 \mathrm{~nm}, 263 \mathrm{~nm}$ and $345 \mathrm{~nm}$ [31]. A weak hypochromicity was observed after CB[7] was annexed to the BER solution. However, an enhancement in absorption spectrum was observed after addition of Hcy into the CB[7]-BER supramolecular system. This should be attributed to the occupation of the $\mathrm{CB}[7]$ hydrophobic cavity by Hcy, giving rise to some BER molecules being expelled.

\section{FT-IR spectroscopy}

The FT-IR spectrum was also analyzed to explore the host-guest interaction between CB[7] and Hcy (Fig. S2). CB[7] has polar carbonyl groups at its two portals and its important representative absorption peak emerges at $1740 \mathrm{~cm}^{-1}$. Hcy has sulfhydryl, methylene and C-S groups, and its special absorption peaks are $2633 \mathrm{~cm}^{-1}$, $2940 \mathrm{~cm}^{-1}$, and $545 \mathrm{~cm}^{-1}$, respectively. Compared with the FT-IR spectrum of CB[7], the carbonyl characteristic peak of $\mathrm{CB}$ [7]-Hcy was blue-shifted to $1732 \mathrm{~cm}^{-1}$. The reason should be the electrostatic interaction between the amino group of Hcy and the carbonyl group of $\mathrm{CB}$ [7] portals. At the same time, the hydrogen of the amino group forms a hydrogen bond with the oxygen of the carbonyl group. In addition, it was observed that both the methylene and the sulfhydryl absorption peaks disappeared, while the C-S characteristic peak weakened in the CB[7]-Hcy system. This indicated that the methylene and sulfhydryl groups of Hcy may have entered the hydrophobic cavity of $\mathrm{CB}[7]$.

\section{Molecular simulation}

Then, the host-guest recognition between $\mathrm{CB}[7]$ and Hcy was explored via theoretical calculations. The CB[7]-Hcy molecular model was optimized at the B3LYP/6-31G(d) level of density functional theory adopting the Gaussian program (Fig. 1). The outcomes verified the partial inclusion of Hcy in the hydrophobic cavity 
of $\mathrm{CB}$ [7]. In the energy-minimized structure $\left(-13057221.14 \mathrm{~kJ} \mathrm{~mol}^{-1}\right)$, the hydrophobic side chain of the Hcy is embedded in the CB[7] macrocyclic molecule cavity, and the amino nitrogen moiety is located in the vicinage of the host carbonyl portal.

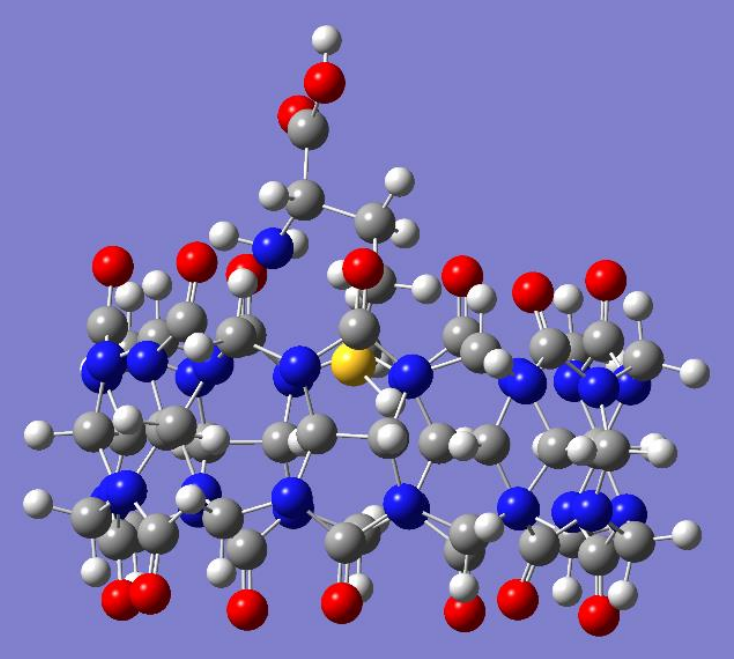

Fig. 1 Energy-minimized structure of the $\mathrm{CB}[7]-\mathrm{Hcy}$ complex in the ground state. Color code for Hcy and CB[7]: oxygen, red; nitrogen, blue; carbon, gray; hydrogen, white; sulfur, yellow

\section{${ }^{1}$ H NMR spectroscopy}

The generation of the CB[7]-Hcy inclusion complex can be further affirmed by ${ }^{1} \mathrm{H}$ NMR spectroscopy (Fig. 2). The consequences obtained are consistent with the results of complex reported in the literature [32]. Compared with the proton signal of the unbound Hcy molecule, the resonance of protons $\mathrm{H} \gamma$ and $\mathrm{H}_{\beta}$ of the bound Hcy in the ${ }^{1} \mathrm{H}$ NMR spectrum of the CB[7]-Hcy system underwent a evident up-field shift, indicating that the hydrophobic cavity of the $\mathrm{CB}$ [7] has a shielding effect on the $-\mathrm{CH}_{2} \mathrm{CH}_{2}$ - of Hcy molecule. Simultaneously, the chemical shift of the resonance hydrogen in the $\mathrm{CB}[7]$ molecule also varied because of the inclusion of Hcy. In short, CB[7] can combine with Hcy to compose host-guest inclusion complexes due to the synergistic hydrophobic effect, hydrogen bonding, ion-dipole interaction, and good size and morphology matching. 


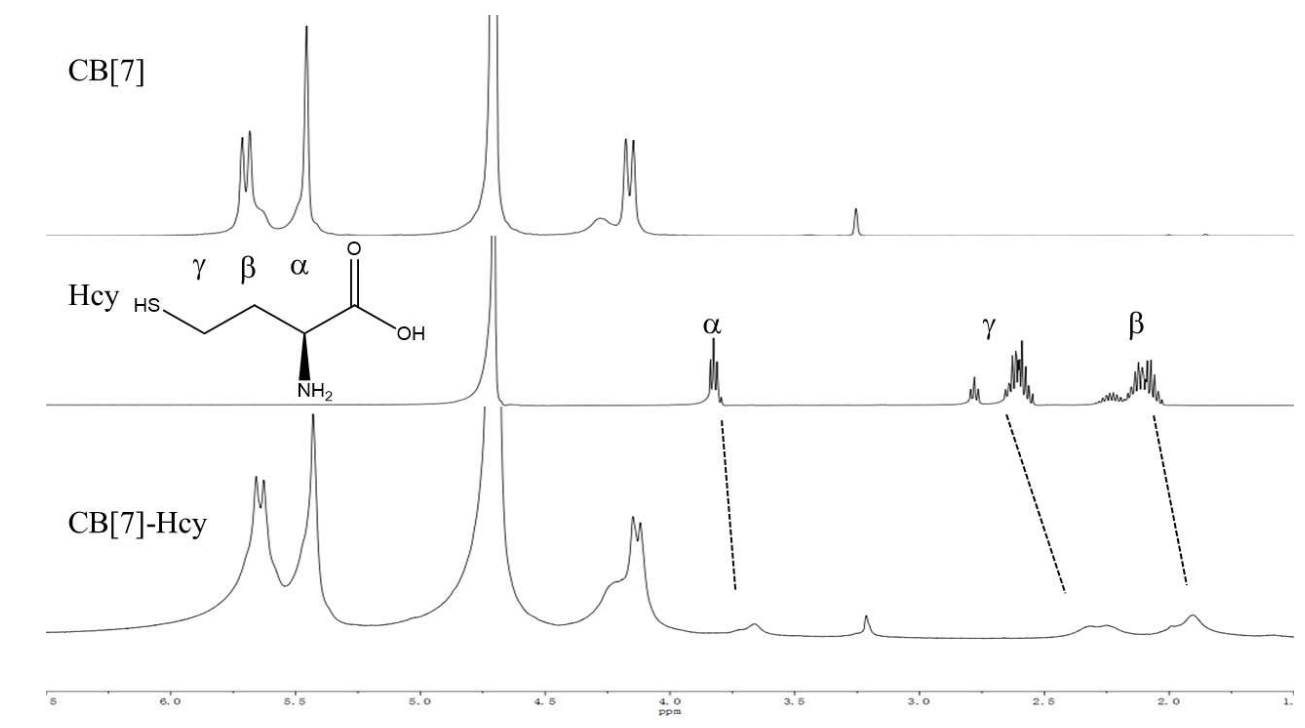

Fig. $2{ }^{1} \mathrm{H}$ NMR spectra (500 MHz) of CB[7], Hcy and CB[7]-Hcy complex

\section{Apparent binding constant and stoichiometry}

The Hcy has no fluorescence in aqueous solution, and there was no obvious change in its UV-vis absorption and fluorescence emission spectra after the addition of $\mathrm{CB}[7]$. Thus, NMR titration and fluorescence spectroscopy was employed to measure the association constant of $\mathrm{CB}$ [7]-Hcy and $\mathrm{CB}$ [7]-BER inclusion complex, respectively $[33,34]$.

For the 1:1 host-guest inclusion complex $(\mathrm{H}-\mathrm{G})$, the fluorescence intensity of the guest (G) was enhanced with the additional non-fluorescent host $(\mathrm{H})$. The fluorescence intensity depends on the concentration of host [28, 35], and the relationship can be expressed by the following equation:

$$
\mathrm{F} / \mathrm{F}_{0}=1+\left(\mathrm{F}_{\infty} / \mathrm{F}_{0}-1\right) \frac{[H] K}{(1+[H] K)}
$$

Where $\mathrm{F}$ represents the fluorescence intensity measured by adding different concentrations of $\mathrm{H}, \mathrm{F}_{0}$ represents the fluorescence intensity of $\mathrm{G}$ in the absence of $\mathrm{H}$, $F_{\infty}$ is the fluorescence intensity when $G$ is completely complexed, and $K$ is the equilibrium binding constant for the $1: 1$ complexation.

$\mathrm{H}+\mathrm{G} \Leftrightarrow \mathrm{H}-\mathrm{G}$

$\mathrm{K}=\frac{[\mathrm{H}-\mathrm{G}]}{[\mathrm{H}][\mathrm{G}]}$

The 1:1 complexation (and, thence, the applicability of Eq. (1)) can be proved from the double reciprocal Benesi-Hildebrand graph of $1 /\left(\mathrm{F}-\mathrm{F}_{0}\right)$ versus $1 /[\mathrm{H}]$. The graph of the 1:1 complex is linear, while the graph of higher-order complexes is nonlinear. 
The equilibrium reaction between $\mathrm{CB}[7]$ and $\mathrm{BER}$ is as follows:

The equilibrium binding constant is expressed as follows:

$\mathrm{K}_{\mathrm{CB}[7]-\mathrm{BER}}=\frac{[\mathrm{CB}[7]-\mathrm{BER}]}{[\mathrm{CB}[7]][\mathrm{BER}]}$

Fig. 3a illustrated the augmentation of BER fluorescence as a function of the addition of $\mathrm{CB}$ [7]. The solid line demonstrated the best fit of the data to Eq. (1) adopting the nonlinear least squares method. Three such tests were carried out, producing the average value of $\mathrm{K}_{\mathrm{CB}[7]-\mathrm{BER}}=1.34 \times 10^{5} \mathrm{~L} \mathrm{~mol}^{-1}$, which is consistent with the corresponding literature result $\left(1.77 \times 10^{5} \mathrm{~L} \mathrm{~mol}^{-1}\right)$ [36]. At the same time, a successive variation Job's plot (Fig. S3) also proved that the CB[7]-BER inclusion complex was constituted with a 1:1 stoichiometry.

The binding affinity between $\mathrm{CB}[7]$ and Hcy was determined by utilizing ${ }^{1} \mathrm{H}$ NMR chemical shift changes of the proton hydrogen resonances after the addition of $\mathrm{CB}[7]$ supramolecular host. The NMR version of the Benesi-Hildebrand equation is expressed as follows [33]:

$1 / \Delta \delta=1 /\left(\mathrm{K}_{\mathrm{a}} \Delta \delta_{\max }\left[\mathrm{H}_{0}\right]\right)+1 / \Delta \delta_{\max }$

where $\Delta \delta$ represents the measured change in chemical shift after adding the host referenced to that of the free guest molecule, $\Delta \delta_{\max }$ represents the discrepancy in chemical shifts between that observed in the guest and in the host-guest inclusion complex, and $\left[\mathrm{H}_{0}\right]$ is the known host concentration.

The production of a 1:1 CB[7]-Hcy host-guest system can be confirmed from the graph of $1 / \Delta \delta$ versus $1 /\left[\mathrm{H}_{0}\right]$ (Fig. 3b). Simultaneously, the calculated binding constant of $\mathrm{K}_{\mathrm{CB}[7]-\mathrm{Hcy}}$ is $2.40 \times 10^{3} \mathrm{~L} \mathrm{~mol}^{-1}$, which is close to the data $(1.09 \pm 0.147) \times 10^{3} \mathrm{~L}$ $\mathrm{mol}^{-1}$ calculated by isothermal titration calorimetry [32]. 

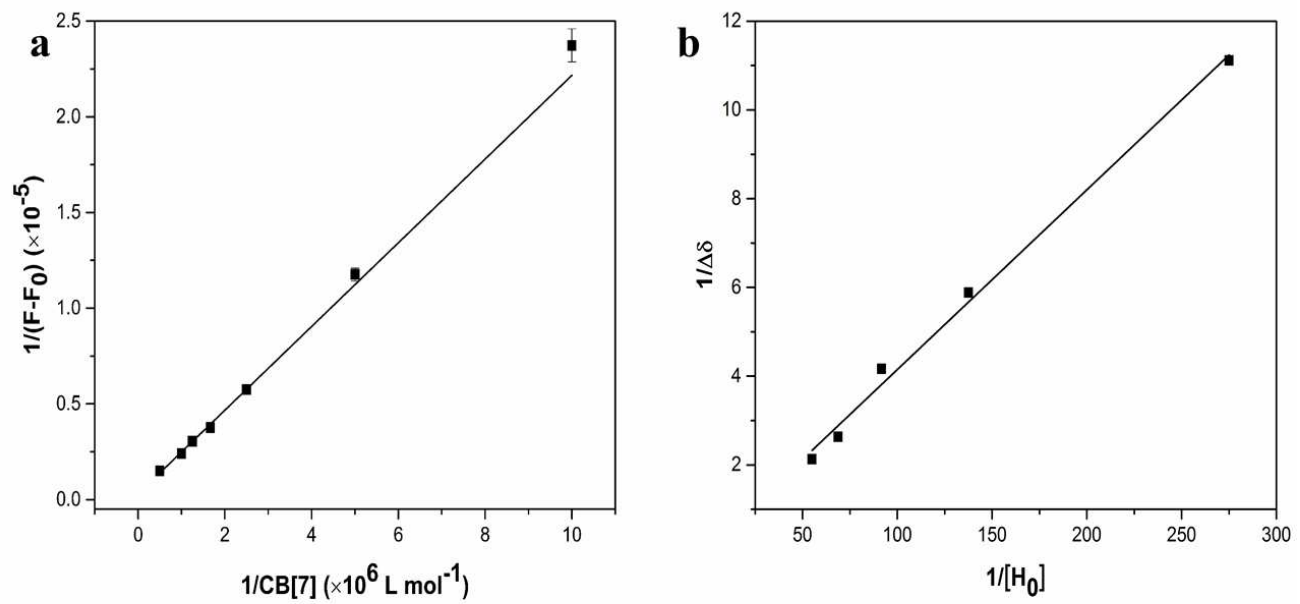

Fig. 3 a: Fluorescence alteration of the $2 \mu \mathrm{M}$ BER solution as a function of the addition of $\mathrm{CB}[7]$ aqueous solution; b: ${ }^{1} \mathrm{H}$ NMR chemical shift alteration of the Hcy solution as a function of the addition of $\mathrm{CB}$ [7] aqueous solution. The inset displayed the linear double reciprocal plot indicating 1:1 inclusion complex. $\mathrm{C}_{\mathrm{CB}[7]}=3 \mu \mathrm{M}$, $\mathrm{C}_{\mathrm{BER}}=2 \mu \mathrm{M}, \lambda_{\mathrm{ex}} / \lambda_{\mathrm{em}}=343 / 500 \mathrm{~nm}$

\section{Response mechanism of the fluorescent probe}

Due to the isoquinoline and substituted benzene rings in BER are not in the same plane, it prevents the formation of a conjugated system. As a result, BER has feeble fluorescence emission in aqueous solution. When $\mathrm{CB}$ [7] was added into the BER solution, the isoquinoline ring part of BER entered the $\mathrm{CB}$ [7] cavity, arousing the electrostatic attraction between the heterocyclic nitrogen of BER and the carbonyl oxygen of the macrocycle. Eventually, the formed complex system leads to the increasing fluorescence of the guest molecule. In addition, the extent of freedom of movement of the BER molecules is reduced, thereby reducing the possibility of radiationless transition [33, 37]. When Hcy was mixed with the CB[7]-BER system, Hcy and BER contended to occupy the hydrophobic cavity of CB[7]. The electrostatic ion-dipole interaction and hydrophobic interaction between the amine group of Hcy and the carbonyl group of $\mathrm{CB}$ [7] portal promote the formation of complexation [27]. The photochemical performance of BER extremely depends on its local microenvironment. Hence, the addition of Hcy makes BER lose the protection of the $\mathrm{CB}$ [7] cavity, causing a reduction in the fluorescence intensity of BER (Scheme 1). 


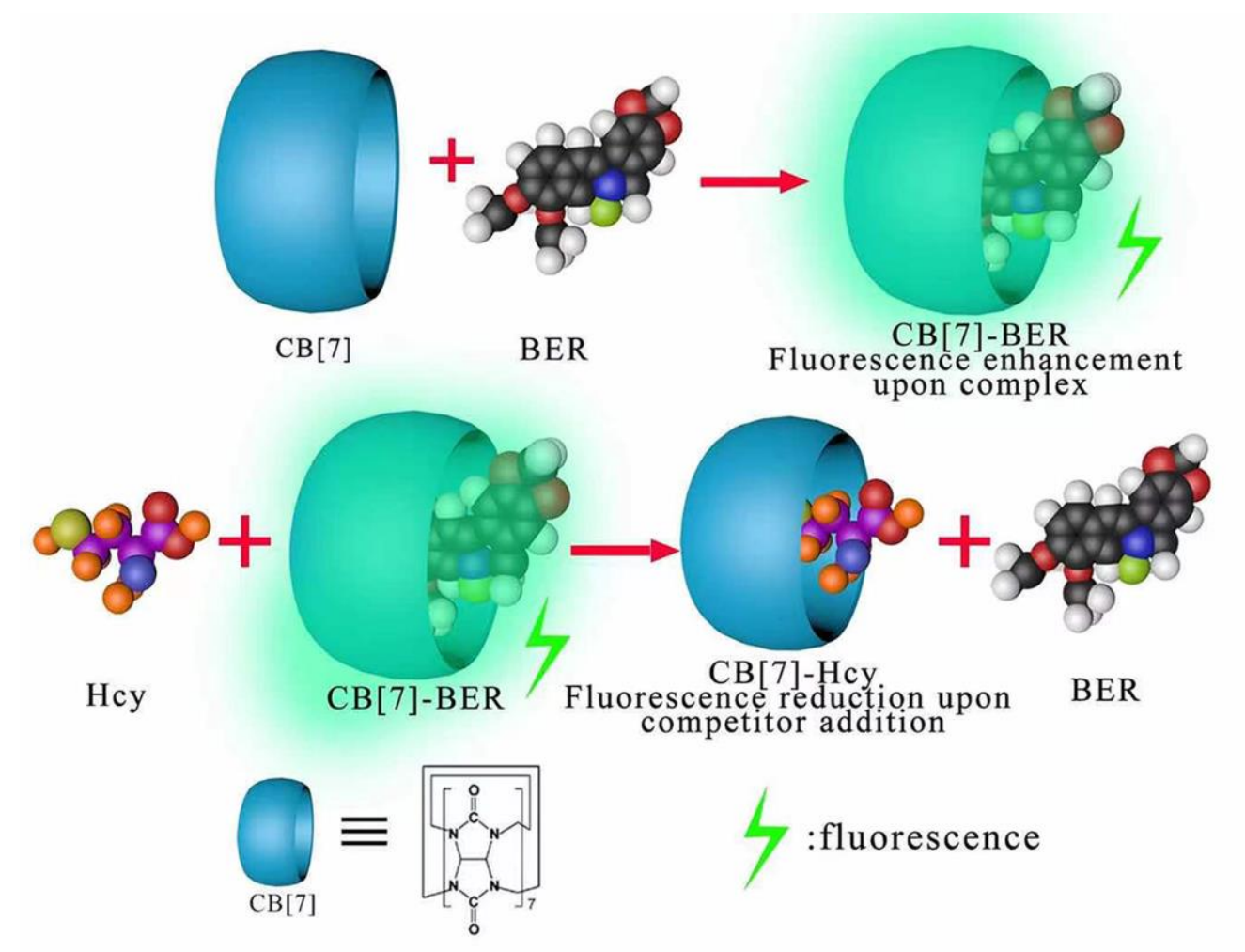

Scheme 1 Competitive recognition of $\mathrm{CB}[7]$ to Hcy against BER Optimization of experimental conditions

Firstly, the influence of changing the BER and $\mathrm{CB}$ [7] concentrations on the fluorescence intensity of the supramolecular system was studied. $\mathrm{CB}[7]$ is spectroscopically inert in aqueous solution [37]. The fluorescence intensity of the $\mathrm{CB}$ [7]-BER complex increased obviously in the range from 1 to $10 \mu \mathrm{M}$ (Fig. S4). In this study, it is extremely important to choose an appropriate BER concentration because it acts as a signal probe. If the concentration of BER is very low, the sensitivity of the probe will also be low. In turn, very high concentration may be detrimental to obtain an optimal detection limit for the analyte. Thus, $2 \mu \mathrm{M}$ was chosen as the optimal BER concentration. Furthermore, the BER aqueous solution itself can only emit weak fluorescence (Fig. S5), but the results showed that the fluorescence intensity was gradually enhanced and then tended to balance after the addition of $3 \mu \mathrm{M} \mathrm{CB}$ [7] (Fig. S6). Therefore, $3 \mu \mathrm{M}$ was chosen as the optimal $\mathrm{CB}$ [7] concentration.

The effect of $\mathrm{pH}$ on $\mathrm{F} / \mathrm{F}_{\mathrm{pH}=7}$ was investigated. When the sample $\mathrm{pH}$ was maintained in the range of 5 to 10 , the fluorescence variation of the system was relatively stable (Fig. S7). However, too high or too low pH was not conducive to the steady change of the fluorescence intensity of the reaction system. Thus, the $\mathrm{pH}$ 
would not be adjusted in subsequent tests.

The impact of temperature on $\mathrm{F} / \mathrm{F}_{0}$ was also researched. As shown in Fig. S8, the formed complexes were stable below $30^{\circ} \mathrm{C}$. $\mathrm{F} / \mathrm{F}_{0}$ weakened greatly when the ambient temperature rose from $30{ }^{\circ} \mathrm{C}$ to $50{ }^{\circ} \mathrm{C}$ in view of the dissociation of the inclusion complexes at higher temperatures. Relying on the above events, all succeeding determinations were carried out at room temperature.

In addition, the outcomes confirmed that $\mathrm{F} / \mathrm{F}_{0}$ reached a minimum quickly within 8 min after the addition of Hcy and kept constant for several hours (Fig S9). Hence, incubation for $8 \mathrm{~min}$ at room temperature was selected as a standard measurement condition.

\section{Analytical performance}

Fig. 4 displayed that the fluorescence intensity of the formed CB[7]-BER complex gradually weakened with the increasing concentration of Hcy. Under the optimized test conditions, the standard calibration graph of $F / F_{0}$ versus Hcy concentration showed a good linear relationship ranging from 5 to $200 \mu \mathrm{M}$. The linear regression equation was $\mathrm{F} / \mathrm{F}_{0}=-0.0003 \mathrm{C}_{\mathrm{Hcy}}+0.8783\left(\mathrm{R}^{2}=0.9999\right)$ with the detection limit (LOD) of $1.76 \mu \mathrm{M}$ ( $\mathrm{LOD}=3 \sigma / \mathrm{S}, \sigma$ : standard deviation, S: slope). Compared with other reported detection methods (Table 1), this established fluorescence method has advantages of fast detection time, satisfactory sensitivity, a wider linear range as well as without sample pretreatment.

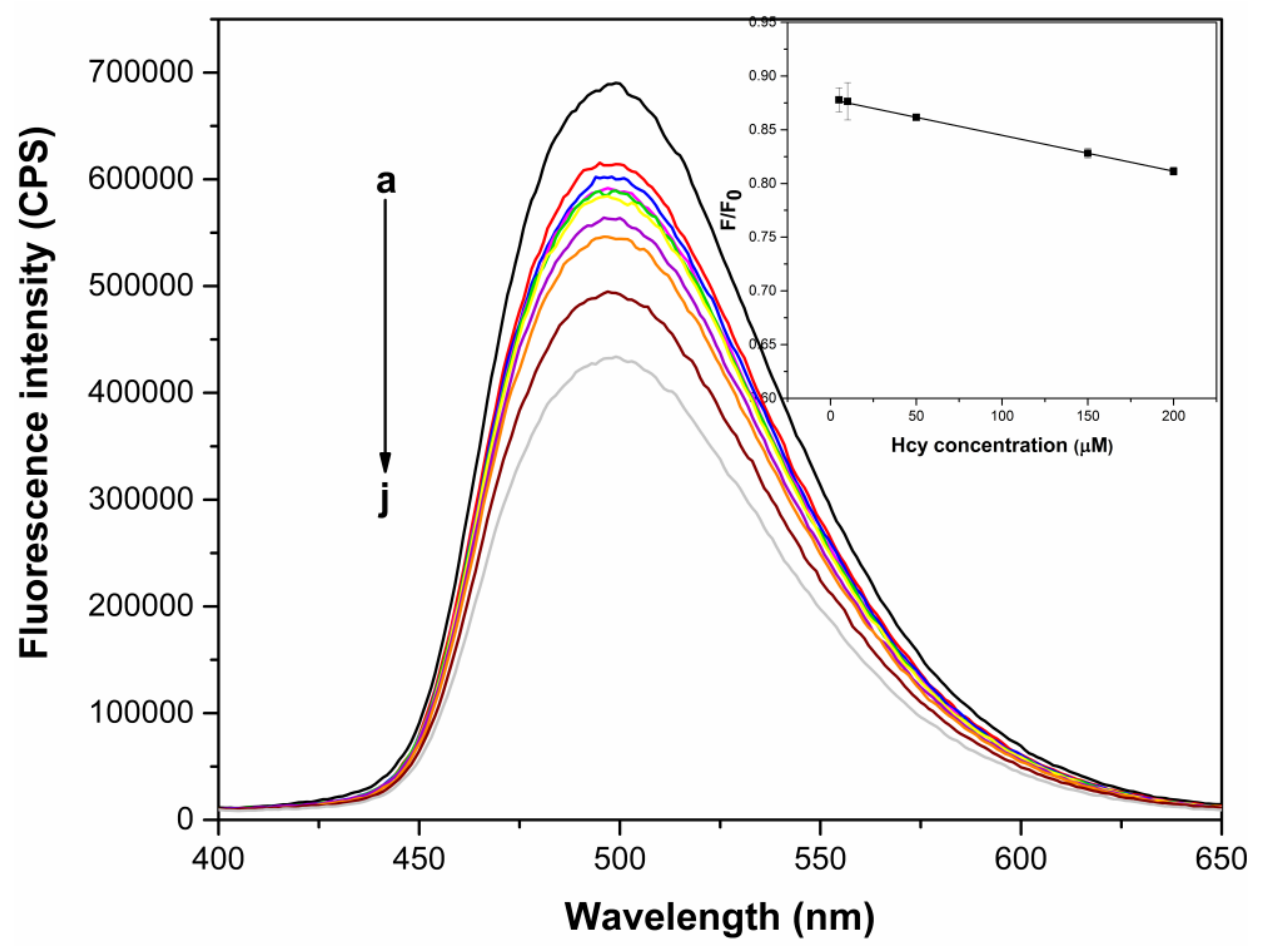


Fig. 4 Fluorescence spectra of the CB[7]-BER system in the existence of dissimilar concentrations of Hcy (from a to j: 0, 1, 5, 10, 50, 80, 100, 200, 500, and $1000 \mu \mathrm{M}$ ). $\mathrm{C}_{\mathrm{CB}[7]}=3 \mu \mathrm{M}, \mathrm{C}_{\mathrm{BER}}=2 \mu \mathrm{M}, \lambda_{\mathrm{ex}}=343 \mathrm{~nm}$. Inset displays the calibration curve of $\mathrm{F} / \mathrm{F}_{0}$ versus Hcy concentration

Table 1 Comparison with other reported technologies for the detection of Hcy

\begin{tabular}{|c|c|c|c|c|c|c|}
\hline $\begin{array}{l}\text { Detection } \\
\text { technique }\end{array}$ & $\begin{array}{c}\text { Sample } \\
\text { pretreatment }\end{array}$ & $\begin{array}{l}\text { Detection } \\
\text { time (min) }\end{array}$ & $\begin{array}{l}\text { Linear } \\
\text { range } \\
(\mu \mathrm{M}) \\
\end{array}$ & $\begin{array}{l}\text { LOD } \\
(\mu \mathrm{M})\end{array}$ & Appliction & Ref. \\
\hline $\begin{array}{c}\text { Colorimetric } \\
\text { probe }\end{array}$ & I & 10 & $2.5-30$ & 2.09 & I & [1] \\
\hline $\begin{array}{l}\text { Differential } \\
\text { pulse } \\
\text { voltammetry }\end{array}$ & $\begin{array}{l}\text { Hcy-binding-a } \\
\text { ptamer was } \\
\text { grafted onto } \\
\text { the surface of } \\
\text { Au } \\
\text { nanoparticles/c } \\
\text { arbon } \\
\text { electrode }\end{array}$ & 60 & $\begin{array}{c}0.05-2 \\
0\end{array}$ & 0.01 & $\begin{array}{l}\text { Human } \\
\text { blood } \\
\text { serum and } \\
\text { urine } \\
\text { samples }\end{array}$ & [2] \\
\hline HPLC-UV & $\begin{array}{c}\text { Precolumn } \\
\text { derivatization }\end{array}$ & 10 & $0.7-10$ & 0.2 & $\begin{array}{l}\text { Pig brain } \\
\text { homogena } \\
\text { tes }\end{array}$ & [8] \\
\hline $\begin{array}{c}\text { Capillary } \\
\text { zone } \\
\text { electrophores } \\
\text { is }\end{array}$ & $\begin{array}{c}\text { Reduction, } \\
\text { derivatization } \\
\text { and capillary } \\
\text { electrophoresis } \\
\text { separation }\end{array}$ & 11.9 & $2-20$ & 0.7 & $\begin{array}{l}\text { Human } \\
\text { plasma } \\
\text { samples }\end{array}$ & [15] \\
\hline $\begin{array}{c}\text { Fluorescence } \\
\text { probe }\end{array}$ & $\begin{array}{l}\text { Modified } \\
\text { carbon dots }\end{array}$ & 15 & $5-50$ & 3.5 & $\begin{array}{l}\text { Human } \\
\text { plasma } \\
\text { samples }\end{array}$ & [38] \\
\hline $\begin{array}{c}\text { Fluorescent } \\
\text { probe }\end{array}$ & Not needed & 8 & $5-200$ & 1.76 & $\begin{array}{l}\text { Bovine } \\
\text { serum }\end{array}$ & $\begin{array}{l}\text { This } \\
\text { work }\end{array}$ \\
\hline
\end{tabular}

The influence of some common interferents on the determination of Hcy was investigated before applying the proposed method to actual sample analysis (Fig. 5). 
The results demonstrated that ions $\left(\mathrm{Na}^{+}, \mathrm{K}^{+}, \mathrm{Ca}^{2+}, \mathrm{HCO}_{3}{ }^{-}, \mathrm{CO}_{3}{ }^{2-}\right)$, amino acids, except high-concentration phenylalanine (alanine, cysteine, valine, methionine, arginine, histidine, lysine, tryptophan), peptides (glutathione) and glucose in serum cannot significantly interfere with the determination of Hcy. Although high concentration of phenylalanine can interfere with the detection of Hcy by the CB[7]-BER probe, the normal concentration of phenylalanine in adult serum is low about $\mu \mathrm{M}$ level, which has little influence in actual serum detection applications. Accordingly, fluorescent probes based on the $\mathrm{CB}[7]$ host have good selectivity for the detection of Hcy.

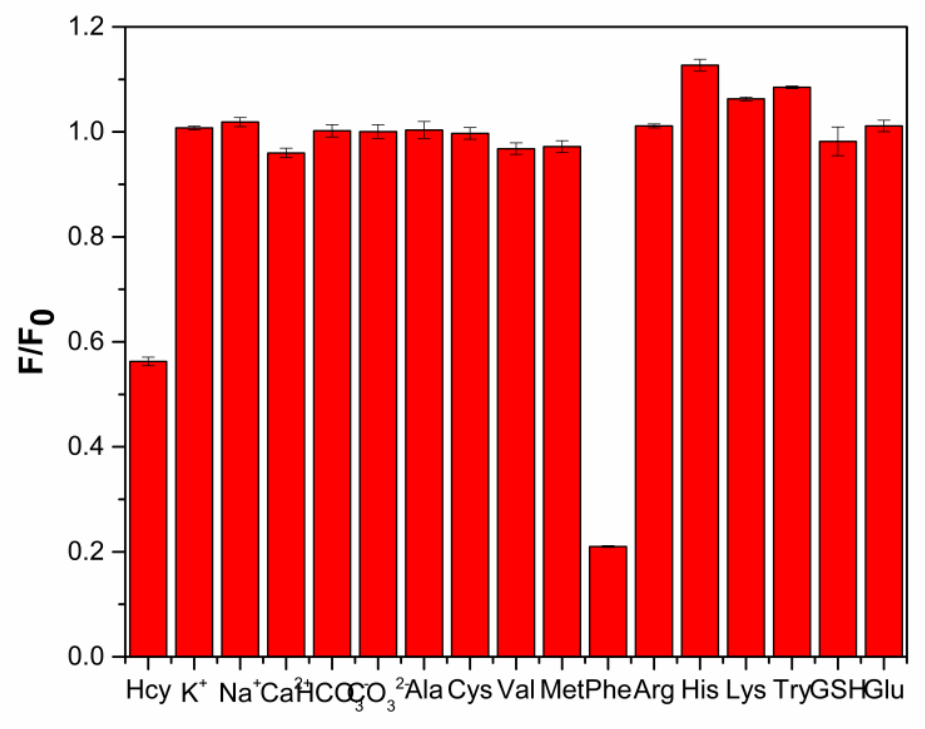

Fig. 5 Influence of interfering substances on the detection of Hcy. The concentration of interferents and Hcy are both $1 \mathrm{mM}$

\section{Analytical application}

In order to verify the practicability of this method, the standard addition method was used to determine Hcy in the real bovine serum samples. From Table 2, the recoveries of examined samples spiked with three different concentrations of Hcy were in the range from $85.1 \%$ to $102.0 \%$, and the related RSDs were lower than $5 \%$. The above analysis results suggested that the fluorescent probe method is effective and reliable, with satisfactory accuracy and precision.

Table 2 Determination of Hcy in spiked serum

\begin{tabular}{ccccc}
\hline Sample & Added $(\mu \mathrm{M})$ & Detected $(\mu \mathrm{M})^{\mathrm{a}}$ & Recovery $(\%)$ & RSD $(\%)$ \\
\hline Bovine serum & 40 & $40.8 \pm 1.1$ & 102.0 & 2.8 \\
& 100 & $98.5 \pm 1.6$ & 98.5 & 1.6 \\
\hline
\end{tabular}




\begin{tabular}{llll}
160 & $136.2 \pm 6.3$ & 85.1 & 4.6 \\
\hline
\end{tabular}

${ }^{\text {a }}$ Mean \pm standard deviation $(\mathrm{n}=3)$

\section{Conclusions}

This work reported a new method for detecting Hcy by means of the host-guest competitive interaction of $\mathrm{CB}[7]$ with Hcy and BER. It was identified that the formation of $\mathrm{CB}$ [7]-BER inclusion complexes strongly enhanced the fluorescence emission of BER because of their powerful coplanarity and rigidity. Because of the host-guest interaction and concentration advantage between CB[7] and Hcy, the BER in the hydrophobic cavity of $\mathrm{CB}$ [7] can be substituted by Hcy, leading to reduce the fluorescence emission of CB[7]-BER complex. This fluorescence probe method based on the competitive strategy was successfully applied to determine Hcy in biological serum, which exhibits good sensitivity and selectivity. The interaction mechanism between the $\mathrm{CB}$ [7] host and the analyte Hcy was also proved that the hydrophobic alkyl chain of Hcy located inside the host through a series of characterization techniques. In conclusion, this supramolecular fluorescent probe provides a promising way for the simple and fast determination of Hcy in clinic.

\section{Acknowledgments}

This work was financially supported by the Natural Science Foundation Projects of China [No. 81950410634]; the Independent Innovation Fund Project of Agricultural Science and Technology of Jiangsu Province in 2017 [No. CX (17) 1003]; the National Innovation and Entrepreneurship Training Program for Undergraduate [No. 202110316029Z]; and the Innovation and Entrepreneurship Training Program for Undergraduate [No. 202110316149].

\section{Author Contributions}

316 Chenrui Jiang: Method establishment, Data analysis, Writing - original draft. Deli Xiao: Supervision, Writing - reviewing \& editing. Ping Yang: Software, Theoretical calculation. Wenyao Tao: Literature investigation. Zhaorui Song: Method establishment, Data analysis. Hua He: Supervision, Funding acquisition, Writing reviewing \& editing.

\section{Data Availability}

The datasets generated during the current study are available from the corresponding author on reasonable request.

Ethics Approval We declare that we have not violated any ethical responsibilities. No 
326 Human Participants and/or Animals were used in this research.

327 Consent to Participate This is not applicable as we have not used any human participants.

329 Consent for Publication This is not applicable as we have not used any human 330 participants.

331 Conflict of Interest The authors declare that they have no confict of interest to the publication of this article.

\section{References}

334 [1] Lin M L, Guo Y J, Liang Z Y et al (2019) Simple and fast determination of biothiols using $\mathrm{Fe} 3+-3$, 3', 5, 5'-tetramethylbenzidine as a colorimetric probe. Microchemical Journal 147:319-323

[2] Beitollahi H, Zaimbashi R, Mahani M T et al. (2020) A label-free aptasensor for highly sensitive detection of homocysteine based on gold nanoparticles. Bioelectrochemistry 134:107497

[3] Chow C F, Lam M H W, Leung M K P (2002) Fluorescent sensing of homocysteine by molecular imprinting. Analytica Chimica Acta 466(1):17-30 [4] Wang H, Mu X, Chen W et al (2021) An ir(iii) complex capable of discriminating homocysteine from cysteine and glutathione with luminescent signal and imaging studies. Talanta 221:121428

[5] Li Y, Liu W, Zhang P et al (2017) A fluorescent probe for the efficient discrimination of cys, hcy and gsh based on different cascade reactions. Biosensors and Bioelectronics 90:117-124

[6] Ding S, Feng G (2016) Smart probe for rapid and simultaneous detection and discrimination of hydrogen sulfide, cysteine/homocysteine, and glutathione. Sensors and Actuators B: Chemical 235:691-697 [7] Park S H, Lee J Y, Cho H N et al (2019) Simple and novel assay of the host-guest complexation of homocysteine with cucurbit[7]uril. Journal of Microbiology and Biotechnology 29(1):114-126

[8] Kamińska A, Olejarz P, Borowczyk K et al (2018) Simultaneous determination of total homocysteine, cysteine, glutathione, and n-acetylcysteine in brain homogenates by HPLC. Journal of Separation Science 41(16):3241-3249 analysis of saliva, plasma and urine for total thiols using HPLC with UV detection. 
[10] Dousa M (2018) The determination of pharmaceutically active thiols using hydrophilic interaction chromatography followed postcolumn derivatization with o-phthaldialdehyde and fluorescence detection. Journal of Pharmaceutical and Biomedical Analysis 156:1-7

[11] Deakova Z, Durackova Z, Armstrong D W et al (2015) Two-dimensional high performance liquid chromatography for determination of homocysteine, methionine and cysteine enantiomers in human serum. Journal of Chromatography A 1408:118-124

[12] Borowczyk K, Chwatko G, Kubalczyk P et al (2016) Simultaneous determination of methionine and homocysteine by on-column derivatization with o-phtaldialdehyde. Talanta 161:917-924

[13] Chang C F, Hamase K, Tsunoda M (2020) Analysis of total thiols in the urine of a cystathionine beta-synthase-deficient mouse model of homocystinuria using hydrophilic interaction chromatography. Molecules 25(7):

[14] Salehzadeh H, Mokhtari B, Nematollahi D (2014) Selective electrochemical determination of homocysteine in the presence of cysteine and glutathione. Electrochimica Acta 123:353-361

[15] Kubalczyk P, Bald E, Furmaniak P et al (2014) Simultaneous determination of total homocysteine and cysteine in human plasma by capillary zone electrophoresis with $\mathrm{pH}$-mediated sample stacking. Analytical Methods 6(12):

[16] Purgat K, Olejarz P, Koska I et al (2020) Determination of homocysteine thiolactone in human urine by capillary zone electrophoresis and single drop microextraction. Analytical Biochemistry 596:113640

[17] Uehara N (2016) Colorimetric assay of homocysteine using gold nanoparticles conjugated with thermoresponsive copolymers. Analytical Methods 8(39):7185-7192 [18] Hu Z, Jiang X, Xu F et al (2016) Colorimetric sensing of bithiols using photocatalytic uio-66(nh2) as h2o2-free peroxidase mimics. Talanta 158:276-282

[19] Yuan C, Qin X, Xu Y et al (2020) Carbon quantum dots originated from chicken blood as peroxidase mimics for colorimetric detection of biothiols. Journal of Photochemistry and Photobiology A: Chemistry 396:

[20] Mohammadi S, Khayatian G (2017) Colorimetric detection of biothiols based on aggregation of chitosan-stabilized silver nanoparticles. Spectrochim Acta A Mol Biomol Spectrosc 185:27-34

[21] Forgacsova A, Galba J, Mojzisova J et al (2019) Ultra-high performance 
hydrophilic interaction liquid chromatography - triple quadrupole tandem mass spectrometry method for determination of cysteine, homocysteine, cysteinyl-glycine and glutathione in rat plasma. Journal of Pharmaceutical and Biomedical Analysis $164: 442-451$

[22] Saylan M, Er E O, Tekin Z et al (2020) An accurate and sensitive analytical method for the simultaneous determination of glycine, methionine and homocysteine in biological matrices by matrix matching strategy and LC-quadrupole-time-of-flight-MS/MS. Spectrochim Acta A Mol Biomol Spectrosc 239:118394

[23] Escobar J, Sanchez-Illana A, Kuligowski J et al (2016) Development of a reliable method based on ultra-performance liquid chromatography coupled to tandem mass spectrometry to measure thiol-associated oxidative stress in whole blood samples. Journal of Pharmaceutical and Biomedical Analysis 123:104-112

[24] Alam S F, Kumar S, Ganguly P (2019) Measurement of homocysteine: A historical perspective. Journal of Clinical Biochemistry and Nutrition 65(3):171-177 [25] Zhu L, Zhao Z, Zhang X et al (2018) A highly selective and strong anti-interference host-guest complex as fluorescent probe for detection of amantadine by indicator displacement assay. Molecules 23(4):

[26] Li C F, Du L M, Wu H et al (2011) Determination of 1-phenylalanine by cucurbit[7]uril sensitized fluorescence quenching method. Chinese Chemical Letters 22(7):851-854

[27] Yang J Y, Du L M, Wu H et al (2011) Determination of 1-cystine by a new sensitive cucurbit[7]uril/palmatine probe. Chinese Journal of Chemistry 29(6):1268-1272

[28] Shi L, Xie J H, Du L M et al (2016) Determination of phenformin hydrochloride employing a sensitive fluorescent probe. Spectrochim Acta A Mol Biomol Spectrosc 162:98-104

[29] Cao Y, Wang S, Wu W et al (2019) Cucurbit[6]uril modified cdte quantum dots fluorescent probe and its selective analysis of p-nitroaniline in environmental samples. Talanta 199:667-673

[30] Li C F, Du L M, Wu W Y et al (2010) Supramolecular interaction of cucurbit[n]urils and coptisine by spectrofluorimetry and its analytical application. Talanta 80(5):1939-1944

[31] Li Y, Li C F, Du L M et al (2015) A competitive strategy based on cucurbit[7]uril 
428 supramolecular interaction for simple and sensitive detection of dibucaine. Talanta

$429 \quad 132: 653-657$

430 [32] Lee M J, Shee N K, Son J-I et al (2019) Supramolecular complexation of 431 homocysteine and cysteine with cucurbit[7]uril. Supramolecular Chemistry

$432 \quad 31(6): 369-376$

433 [33] Wang G Q, Guo L, Du L M et al (2013) Competitive supramolecular interaction 434 of carbachol and berberine with cucurbit[7]uril and its analytical application.

435 Microchemical Journal 110:285-291

436 [34] Wang G Q, Du L M, Guo Y H et al (2013) Study on the supramolecular 437 interaction of astemizole with cucurbit[7]uril and its analytical application. Anal. 438 Methods 5(1):173-179

439 [35] Jing X, Du L M, Wu H et al (2012) Determination of pesticide residue cartap 440 using a sensitive fluorescent probe. Journal of Integrative Agriculture 441 11(11):1861-1870

442 [36] Li C, Feng J, Ju H (2015) Supramolecular interaction of labetalol with 443 cucurbit[7]uril for its sensitive fluorescence detection. Analyst 140(1):230-235

444 [37] Dong N, Cheng L N, Wang X L et al (2011) Significant fluorescence 445 enhancement by supramolecular complex formation between berberine chloride and 446 cucurbit(n=7)uril and its analytical application. Talanta 84(3):684-689

447 [38] Zhu H, Wang E, Li J et al (2018) L-tyrosine methyl ester-stabilized carbon dots 448 as fluorescent probes for the assays of biothiols. Analytica Chimica Acta 1006:83-89 


\section{Supplementary Files}

This is a list of supplementary files associated with this preprint. Click to download.

- Fig.S1.tif

- Fig.S2.tif

- Fig.S9.tif

- Fig.S3.tif

- Fig.S4.tif

- Fig.S5.tif

- Fig.S6.tif

- Fig.S7.tif

- Fig.S8.tif

- Scheme1.jpg 